\title{
Vapor Phase Crystal Growth of Zinc Selenide under Controlled Partial Pressure and Its Crystal Structure*
}

\author{
By Teruo Kiyosawa**, Kenzo Igaki*** \\ and Nobumitsu $\bar{O}$ hashi***
}

\begin{abstract}
Zinc selenide single crystals were grown by the sublimation method under variously controlled partial pressures of one of the constituent elements using a closed quartz ampule with the pure $\mathrm{Zn}$ or Se reservoir at one end.

The relation between the partial pressure of $\mathrm{Zn}$ or $\mathrm{Se}_{2}$, which was estimated from the reservoir temperature, and the transport rate of solid $\mathrm{ZnSe}$ from the charged source to the growth chamber via the vapor phase was found as follows :

(1) In the case of low partial pressure of $\mathrm{Zn}$ or $\mathrm{Se}_{2}$, i. e. low reservoir temperature, the transport rate was independent of partial pressure.

(2) In the case of high $\mathrm{Zn}$ partial pressure, it was proportional to the $-3 \mathrm{rd}$ power of $\mathrm{Zn}$ partial pressure.

(3) For the high $\mathrm{Se}_{2}$ partial pressure, it was proportional to the $-3 / 2$ th power of $\mathrm{Se}_{2}$ partial pressure.

These experimental results were compared with the calculated results which were obtained on the assumption that the rate determining procedure for the transport process was the diffusion in the vapor phase, and partial pressure regions showing good agreement between the experimental and calculated results were disclosed.

The grown crystals had always a crystal structure of the zincblende type and their typical shapes were columnar with an equilateral hexagonal cross section grown in one of the $\langle 111\rangle$ directions and also plate-like grown in two of the $<111\rangle$ directions.

$\mathrm{X}$-ray diffraction photographs by the oscillating crystal method and the Weissenberg method revealed two types of twin structures caused by growth faults; one due to the growth faults in only one of the $<111>$ directions which corresponded to the growth direction of the columnar crystals and the other due to the growth faults in at least two of the $<111>$ directions which corresponded to growth directions of the plate-like crystals.

(Received November 18, 1971)
\end{abstract}

\section{Introduction}

The II and the VI group elements form semiconducting compounds of a type $A^{I I} B^{\text {VI }}$ which show remarkable properties such as photoconductivity.

These compounds have relatively high melting points and appreciable dissociation vapor pressures at their melting points, so that the vapor phase reactions are often used for the single crystal growth of these compounds because of their relatively low operating temperature. One of the vapor phase crystal growth methods is the sublimation method developed by Czyzak et al. ${ }^{(1)}$ and modified by Greene et al. (2) and Piper et al. (3) A unique method was arranged by Koňák et al. ${ }^{(4)}$ and Prior $^{(5)}$ which made possible to grow crystals under controlled partial pressure of one of the constituent elements using a reservoir of one of the constituent elements.

In the present study the method of Koňák et al. and Prior was modified and applied to the growth of $\mathrm{ZnSe}$ single crystals with controlled deviation from stoichiometry. The experimental conditions for preparing single crystals and the effectiveness of this method to

* The original of this paper, written in Japanese was published previously in J. Japan Inst. Metals, 34 (1970), 1194.

** Graduate School, Tohoku University, Sendai. Present address: National Research Institute for Metals, Tokyo, Japan.

*** Department of Materials Science, Faculty of Engineering, Tohoku University, Sendai, Japan.

(1) S. J. Czyzak et al. : J. Appl. Phys., 23 (1952), 932.

(2) L. C. Greene et al. : J. Chem. Phys., 29 (1958), 1375.

Trans. J I M control the deviation from stoichiometry were discussed. The crystallographic defects of the grown crystals were also examined.

\section{Experimental Procedures}

\section{Synthesis of $\mathrm{ZnSe}$ polycrystals}

After 1/20 moles of stoichiometric mixture of high purity $\mathrm{Zn}$ and Se was weighed and introduced into a quartz ampule of $10 \mathrm{~mm} \phi \times 80 \mathrm{~mm}$, the ampule was first evacuated, filled with high purity argon gas $(99.99 \%)$ after the gas was exchanged several times, and finally sealed off at the pressure of $10^{-3} \mathrm{mmHg}$. The ampules were then kept at $1000^{\circ} \mathrm{C}$ for 3 to 4 days in a horizontal electric furnace and cooled slowly in the furnace down to room temperature.

\section{Growth of ZnSe single crystals}

A schematic construction of the electric furnace and an example of a temperature profile used to grow $\mathrm{ZnSe}$ single crystals are shown in Fig. 1. The fused quartz ampule consisted of : (a) the source chamber containing ZnSe polycrystals crushed to about 1 to $3 \mathrm{~mm}$ particle size, (b) the $\mathrm{Zn}$ or Se reservoir containing high purity $\mathrm{Zn}$ or Se and (c) the growth chamber where $\mathrm{ZnSe}$ single crystals were grown. The ampule was vacuum sealed in the same way as previously described. The growth chamber was baked out before introducing the ampule

(3) W. W. Piper and S. J. Polich : J. Appl. Phys., 32(1961), 1278.

(4) P. Höschl and Č. Koňák : phys. stat. sol., 9 (1965), 167.

5 A. C. Prior : J. Electrochem. Soc., 108 (1961), 82.

1972 Vol. 13 


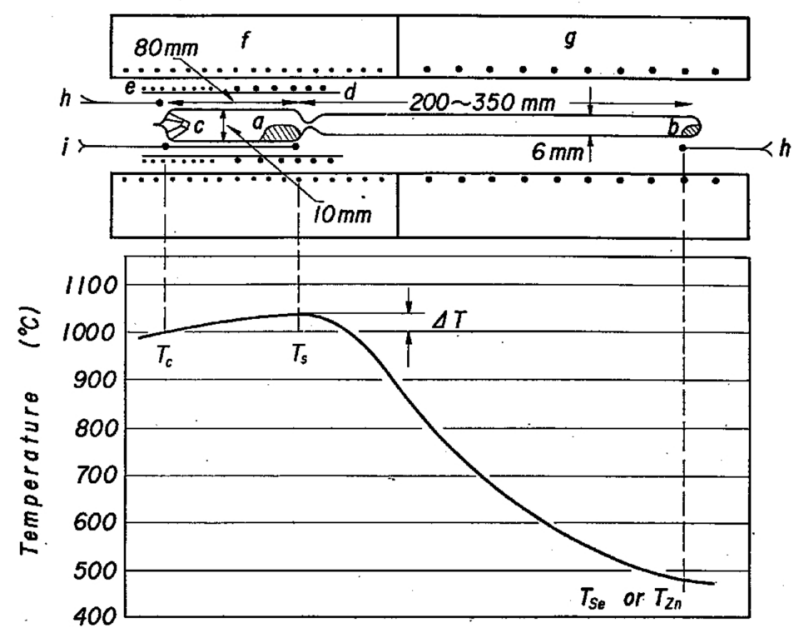

Fig. 1 Schematic construction of the electric furnace and an example of temperature profile.

(a) source chamber (b) $\mathrm{Zn}$ or Se chamber (c) growth chamber (d), (e) auxiliary heater (f), (g) main heater (h) Pt-Pt. Rh thermocouple (i) Pt-Pt. Rh differential thermocouple

into the electric furnace, in order to clean the inner wall.

In the following, the temperatures of the source chamber, the growth chamber and the Zn or Se reservoir are represented as $T_{s}, T_{c}$ and $T_{\mathrm{Zn}}$ or $T_{\mathrm{Se}}$, respectively. The temperature difference $\Delta T$ between $T_{s}$ and $T_{c}$ was detected by a $\mathrm{Pt}-\mathrm{Pt} \cdot \mathrm{Rh}$ differential thermocouple and regulated by two auxiliary heaters. In this study the experimental conditions of $T_{s}=1015^{\circ} \mathrm{C}, \Delta T=15^{\circ} \mathrm{C}$ and $T_{s}=1115^{\circ} \mathrm{C}, \Delta T=15^{\circ} \mathrm{C}$ were employed. The partial vapor pressure control was performed by assuming that the vapor pressure of $\mathrm{Zn}$ or $\mathrm{Se}_{2}$ in the ampule was determined by the equilibrium vapor pressure of $\mathrm{Zn}$ or Se at $T_{\mathrm{zn}}$ or $T_{\mathrm{Se}}$.

After the crystal growth lasted for 1 to 10 days depending upon the transport rates, the ampule was water-quenched and the crystals grown at the growth chamber were weighed to calculate the transport rate.

\section{Crystallographic study}

Specimens 0.2 to $0.4 \mathrm{~mm}$ in thickness for the crystallographic study were cleaved out from the grown single crystals and examined by the oscillating crystal method and the Weissenberg method.

\section{Experimental Results}

A well-grown single crystal is shown in Photo. 1. Its

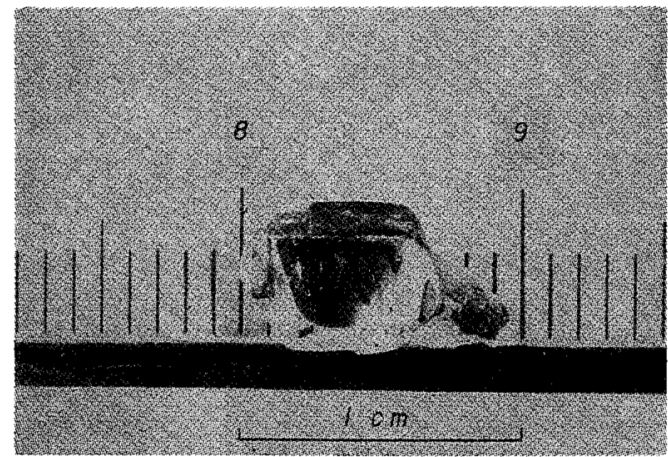

Photo. 1 A well-grown single crystal. shape is columnar with a nearly equilateral hexagonal cross section. X-ray diffractometry revealed that the six columnar planes were $\{110\}$ planes and the growth direction (longitudinal direction) was the $\langle 111\rangle$ direction. Most of the columnar crystals had the dimension of 3 to $5 \mathrm{~mm}$ length and 1 to $2 \mathrm{~mm}$ thickness. In several cases, some or many single crystals joined together into a conical cluster which grew along the wall of the conical tip of the growth chamber. Figures 2 and 3 show the experimental results of the transport rate of solid ZnSe from the charged source to the growth chamber via the vapor phase under the condition of $T_{s}=1015^{\circ} \mathrm{C}$ with $\Delta T=15^{\circ} \mathrm{C}$ or $T_{s}=1115^{\circ} \mathrm{C}$ with $\Delta T=15^{\circ} \mathrm{C}$ and of varied $T_{\mathrm{Zn}}$ or $T_{\mathrm{Se}^{\cdot}}$. The results are summarized in a form of the relation between the transport rate and $\mathrm{Zn}$ or $\mathrm{Se}_{2}$ partial pressure corresponding to $T_{\mathrm{zn}}$ or $T_{\mathrm{Se}}$.

The relation between the transport rate and $\mathrm{Zn}$ partial pressure $^{(6)}$ which is calculated from $T_{\mathrm{Zn}}$ for the $\mathrm{Zn}$ reservoir and that between the transport rate and $\mathrm{Se}_{2}$ partial pressure ${ }^{(6)}$ which is calculated from $T_{\mathrm{Se}}$ for the Se reservoir are shown in Figs. 2 and 3, respectively. From these results the following relations between the partial pressure of $\mathrm{Zn}$ or $\mathrm{Se}_{2}$ and the transport rate were obtained : (1) In the case of a low partial pressure of $\mathrm{Zn}$

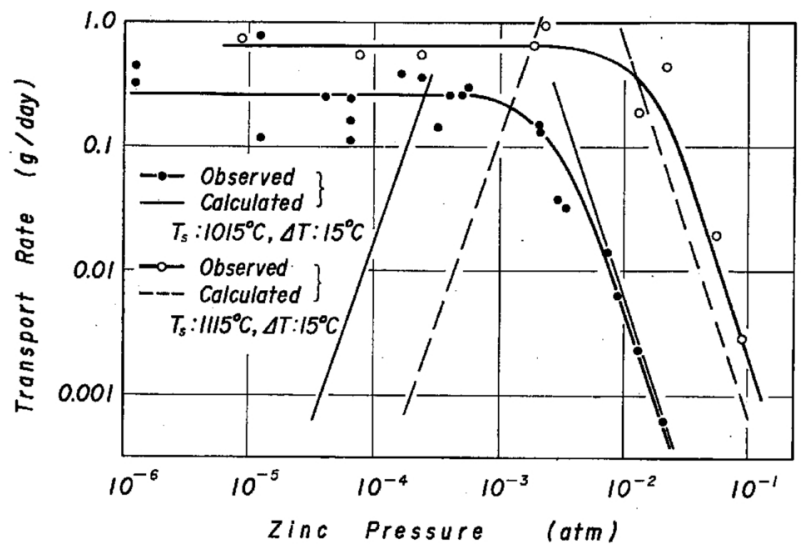

Fig. 2 Observed and calculated relation between transport rate and partial pressure of $\mathbf{Z n}$.

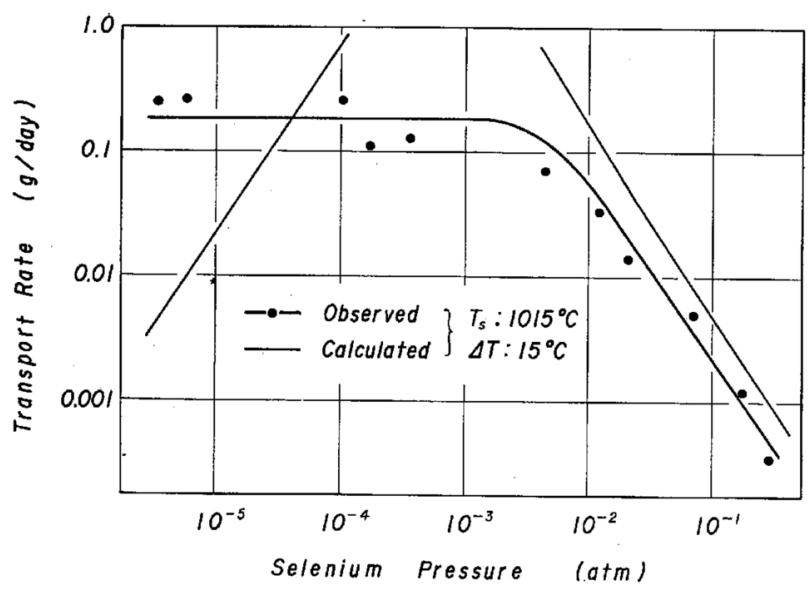

Fig. 3 Observed and calculated relation between transport rate and partial pressure of $\mathrm{Se}_{2}$.

(6) A. N. Nesmeyanov: Vapor Pressure of the Chemical Elements, Elsevier Publishing Co., Amsterdam, (1963), p. 189, 322. 
or $\mathrm{Se}_{2}$, i. e. a low reservoir temperature, the transport rate is independent of the partial pressure. (2) In the case of a high $\mathrm{Zn}$ partial pressure, it is proportional to the -3 rd power of $\mathrm{Zn}$ partial pressure. (3) For the high $\mathrm{Se}_{2}$ partial pressure, it is proportional to the $-3 / 2$ th power of $\mathrm{Se}_{2}$ partial pressure.

The following results were obtained from oscillating crystal and Weissenberg X-ray diffraction photographs of single crystals grown under various growth conditions.

(1) All the specimens have a crystal structure of the zinc blende type with lattice constant $a=5.67 \mathrm{~A}$.

(2) There are two types of twin structure caused by growth faults.

Oscillating crystal X-ray diffraction photographs of the specimens cleaved along the ( $1 \overline{1} 0)$ plane of columnar crystals and oscillated about the $[111]$ direction corresponding to the growth direction as shown in Fig. 4 (a) presented a diffraction pattern with a feature

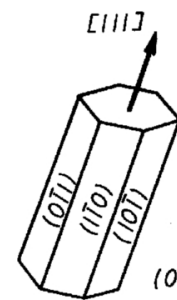

(a)

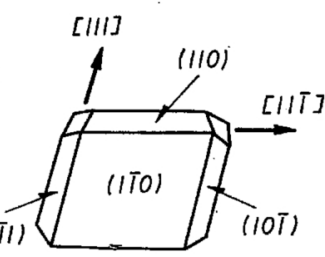

(b)
Fig. 4 Schematic representation of typical crystal habits of vapor grown crystals.
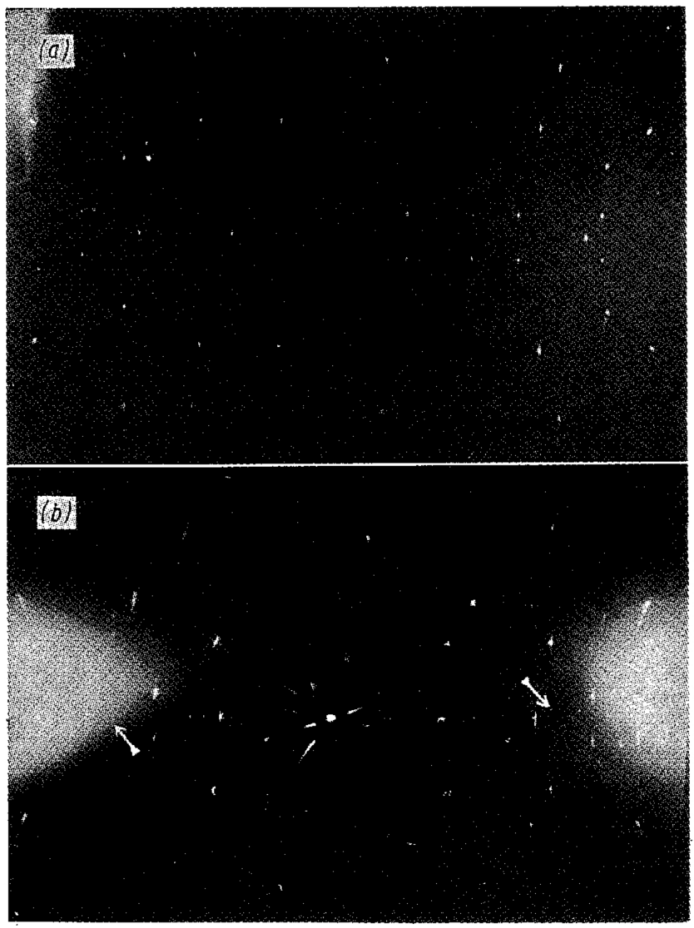

Photo. 2 Oscillation photographs

(a) Obtained from columnar-shaped crystal. Oscillation axis [111]; growth direction. Oscillation angle $30^{\circ}$

(b) Obtained from plate-like crystal. Oscillation axis $[11 \overline{1}]$; one of growth directions.

Oscillation angle $30^{\circ}$

Arrows denote extra $n / 3$ layer spots. characteristic of growth faults such as AaBbCcAa $\mathrm{BbAaCcBbAa} \cdots(\mathrm{A}, \mathrm{B}, \mathrm{C}$ are atomic planes of $\mathrm{Se}$, and $\mathrm{a}$, $\mathrm{b}, \mathrm{c}$ are atomic planes of $\mathrm{Zn})^{(7)}$ occurring in the growth direction (oscillation axis direction). As indicated in Photo. 2 (a) a diffraction pattern symmetric concerning 0 -th layer like the wurtzite type was observed.

On the other hand, specimens from plate-like crystals shown in Fig. 4 (b) which resulted from growing in two of the $\langle 111\rangle$ directions such as [111] and [III], cleaved and oscillated in the same crystallographic orientation as above gave another diffraction pattern which had extra diffraction spots on $n / 3 \operatorname{th}(n=3 m \pm 1)$ layers in addition to the symmetry about 0 -th layer already described. This is shown in Photo. 2 (b).

The distribution of these extra spots in the reciprocal lattice space is determined by the Weissenberg method. The result is shown in Fig. 5 . Figure 5 (a) represents the reciprocal lattice of normal $f c c$ lattice. Extra spots exist only on the (I10) reciprocal lattice planes such as $\mathrm{OABC}$ and DEFG planes. Figure $5(\mathrm{~b})$ is a projection of these spots on $\mathrm{OABC}$ plane.

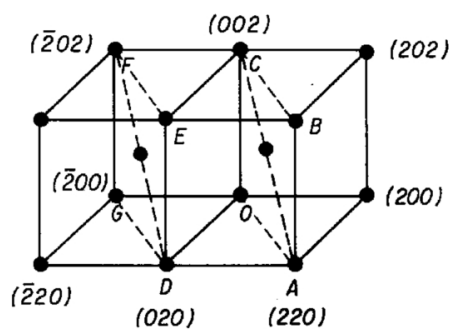

(a)

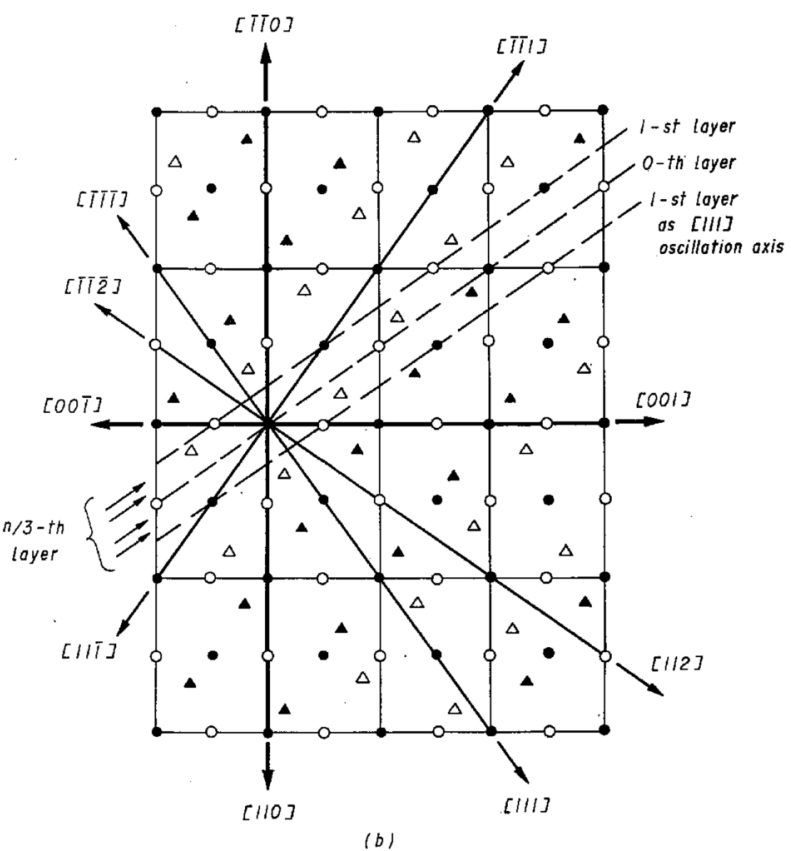

Fig. 5 (a) Reciprocal lattice of $f c c$ structure.

(b) Reciprocal lattice representation of extra $n / 3$ layer spots in Photo. 2 (b) projected on OABC plane.

0,0 :Normal spots on OABC and DEFG planes respectively.

$\Delta, \triangle:$ Extra $n / 3$ layer spots on OABC and DEFG planes respectively.

(7) M. S. Patterson : J. Appl. Phys., 23 (1952), 805. 
It can be seen from Fig. 5 (b) that the extra spots are distributed symmetrically in regard to the [112] direction and, therefore, these extra spots on $n / 3$ th layers can be explained as caused by the twin structure occurring in the [11] ] direction.

It becomes clear that in the plate-like crystals the twin structure is brought about in two of the four equivalent $<111>$ directions.

Conclusively speaking, two types of twin structure due to growth faults are found one is that occurring in only one $<111\rangle$ direction, and the other is that occurring in at least two of the four equivalent $<111>$ directions.

The relation between the type of the twin structure and the crystal growth conditions such as $T_{s}, T_{c}, T_{\mathrm{zn}}$ and $T_{\mathrm{Se}}$ is not clear.

\section{Discussion on Transport Rate}

In this section, the relation between the transport rate and $\mathrm{Zn}$ or $\mathrm{Se}_{2}$ partial pressure will be discussed. Since the single crystal growth method used in this study has the transport rate of 1 to $500 \mathrm{mg} /$ day and operates in a closed ampule, the following assumptions for the situation inside the ampule may be reasonable.

(1) The establishment of the solid-vapor equilibrium in the $\mathrm{Zn}$ or Se reservoir, the source chamber and the growth chamber is rapid enough for a steady state to be realized. Therefore, the sublimation and condensation do not act as a rate determining process.

(2) Total vapor pressure $P$ is constant anywhere in the ampule.

Under these circumstances, the rate determining process for the transport rate may be a diffusion process caused by the difference in the vapor phase composition between the source chamber and the growth chamber. The mass transport phenomenon in a gas mixture is described by kinetic theory of gas molecule as follows ${ }^{(8)}$ :

The mass average velocity $v_{0}$ is defined as

$$
v_{0}=\Sigma n_{i} m_{i} \bar{v}_{i} / \rho,
$$

where $n_{i}, m_{i}$ and $\bar{v}_{i}$ is the number of gas molecule per unit volume, the molecular weight and the mean velocity of $i$-th molecular species, respectively. $\rho$ is the density of a gas mixture and is expressed as

$$
\rho=\Sigma n_{i} m_{i}
$$

Diffusion velocity $V_{i}$ of $i$-th molecular species is defined as the one relative to the mass average velocity $v_{0}$, and

$$
V_{i}=\vec{v}_{i}-v_{0} \text {. }
$$

If the thermal diffusion term can be neglected, $V_{i}$ can also be written as follows:

$$
\begin{aligned}
V_{i}= & \left(n^{2} / n_{i} \rho\right) \sum_{j} m_{j} D_{i j}\left[\partial\left(n_{j} / n\right) / \partial x+\right. \\
& \left.\left\{\left(n_{j} / n\right)-\left(n_{j} m_{j} / \rho\right)\right\} \partial \ln P / \partial x\right],
\end{aligned}
$$

where $n=\Sigma n_{i}, P=\Sigma P_{i}$ and $D_{i j}$ is the multicomponent diffusion coefficient.

(8) J. O. Hirschfelder, C.F. Curtiss and R.B. Bird : Molecular Theory of Gases and Liquids, John Wiley and Sons, (1964), p. $453,516,544$.
If a two components gas mixture has no total pressure difference over the whole region of a closed ampule, eq. (4) can be rewritten as

$$
\begin{aligned}
& V_{1}=\left(n^{2} / n_{1} \rho\right) m_{2} D_{12} \partial\left(n_{2} / n\right) / \partial x \\
& \text { and } \quad V_{2}=\left(n^{2} / n_{2} \rho\right) m_{1} D_{21} \partial\left(n_{1} / n\right) / \partial x \text {. }
\end{aligned}
$$

Further,

$$
\begin{gathered}
D_{12}=D_{21} \\
\text { and } \quad \partial\left(n_{2} / n\right) / \partial x=-\partial\left(n_{1} / n\right) / \partial x
\end{gathered}
$$

where $D_{12}$ and $D_{21}$ are the diffusion coefficients of the two-component gas mixture.

In the present experiments the mass transport corresponding to the mass average velocity must result in the formation of solid $\mathrm{ZnSe}$ crystals by condensation at the growth chamber temperature $T_{c^{*}}$. Therefore, if suffices 1 and 2 represent the $\mathrm{Zn}$ gas molecule and the $\mathrm{Se}_{2}$ gas molecule, respectively, the following relation must be fulfilled :

$$
n_{1} \bar{v}_{1}=2 n_{2} \bar{v}_{2}
$$

Then eq. (1) is rewritten as

$$
\begin{aligned}
v_{0} & =n_{1} \bar{v}_{1}\left\{m_{1}+\left(m_{2} / 2\right)\right\} / \rho \\
& =n_{2} \bar{v}_{2}\left\{2 m_{1}+m_{2}\right\} / \rho .
\end{aligned}
$$

From eqs. (3) and (10) and eq. (5) or (6), $\bar{v}_{1}$ and $\vec{v}_{2}$ can be derived and presented by the following equations neglecting the small variation of $n$ along the temperature gradient between $T_{s}$ and $T_{c}$

$$
\begin{aligned}
& \bar{v}_{1}=\left[n / n_{1}\left\{n_{2}-\left(n_{1} / 2\right)\right\}\right] D_{12} \partial n_{2} / \partial x \\
& \bar{v}_{2}=\left[n / n_{2}\left\{n_{1}-2 n_{2}\right\}\right] D_{21} \partial n_{1} / \partial x .
\end{aligned}
$$

Using these relations, the transport rate of $\mathrm{ZnSe}, \Psi_{\mathrm{ZnSe}}$ $\left(\mathrm{g} / \mathrm{cm}^{2} \cdot \mathrm{sec}\right)$, is expressed as

$$
\begin{gathered}
\Psi_{\mathrm{ZnSe}}=W n_{1} \bar{v}_{1} / N_{0}=\left[W n / N_{0}\left\{n_{2}-\left(n_{1} / 2\right)\right\}\right] D_{12} \partial n_{2} / \partial x \\
=2 W n_{2} \bar{v}_{2} / N_{0}=\left[W n / N_{0}\left\{\left(n_{1} / 2\right)-n_{2}\right\}\right] D_{21} \partial n_{1} / \partial x,
\end{gathered}
$$

where $N_{0}$ is the Avogadro number, $W$ is the molecular weight of $\mathrm{ZnSe}$. According to the kinetic theory of gas molecule, $D_{12}=D_{21}$ is given by $2.628 \times 10^{-3}\left[T^{\prime 3}\right.$ $\left.\left(M_{1}+M_{2}\right) / 2 M_{1} M_{2}\right]^{1 / 2} / P\left\{\left(\sigma_{1}+\sigma_{2}\right) / 2\right\}^{2}=A / P$, where $A=$ $2.628 \times 10^{-3}\left[T^{\prime 3}\left(M_{1}+M_{2}\right) / 2 M_{1} M_{2}\right]^{1 / 2} /\left\{\left(\sigma_{1}+\sigma_{2}\right) / 2\right\}^{2}$, $T^{\prime}=\left(T_{s}+T_{c}\right) / 2$,

$\sigma$ is the molecular diameter (in $A$ unit), $P$ is the total pressure in atm., and $M_{1}$ and $M_{2}$ are the molecular weights of $\mathrm{Zn}$ and $\mathrm{Se}_{2}$ gas molecule, respectively. Substituting $A / P$ for $D_{12}=D_{21}$ in eqs. (13) and (14) and converting $n_{1}$ and $n_{2}$ into the pressure expression, eqs. (13) and (14) become

$$
\begin{aligned}
\Psi_{\mathrm{ZnSe}} & =\left[W A / N_{0} k T^{\prime}\left\{P_{2}-\left(P_{1} / 2\right)\right\}\right] \partial P_{2} / \partial x \\
& =A^{\prime}\left(\partial P_{2} / \partial x\right) /\left\{P_{2}-\left(P_{1} / 2\right)\right\} \\
& =\left[W A / N_{0} k T^{\prime}\left\{\left(P_{1} / 2\right)-P_{2}\right\}\right] \partial P_{1} / \partial x \\
& =A^{\prime}\left(\partial P_{1} / \partial x\right) /\left\{\left(P_{1} / 2\right)-P_{2}\right\},
\end{aligned}
$$

where $A^{\prime}=A W \mid N_{0} k T^{\prime}$ and $k$ is the Boltzman constant. 
Next, $\partial P_{1} / \partial x=-\partial P_{2} / \partial x$ is derived as follows: The solid-vapor equilibrium of $\mathrm{ZnSe}$ is given by ${ }^{(9)}$

$$
2 \mathrm{ZnSe}(s) \rightleftarrows 2 \mathrm{Zn}(g)+\mathrm{Se}_{2}(g)
$$

and $\log K_{p}(T)=\log P_{\mathrm{Zn}_{\mathrm{n}}}^{2} P_{\mathrm{Se}_{2}}=(-37067 / T)+19.4$.

Since the total pressure $P$ is a function of partial pressure $P_{\mathrm{zn}}$ or $P_{\mathrm{Se}_{2}}$ determined by $T_{\mathrm{zn}}$ or $T_{\mathrm{Se}}$, respectively,

for the $\mathrm{Zn}$ reservoir,

$$
P=P_{\mathrm{zn}_{\mathrm{n}}}+P_{\mathrm{Se}_{2}}=P_{\mathrm{zn}_{\mathrm{n}}}+\left(K_{p} / P_{\mathrm{Zn}_{\mathrm{n}}}^{2}\right)
$$

for the Se reservoir,

$$
P=P_{\mathrm{zn}}+P_{\mathrm{Se}_{2}}=\left(K_{p} / P_{\mathrm{Se}_{2}}\right)^{1 / 2}+P_{\mathrm{Se}_{2}} .
$$

Figure 6 shows these relations at $T_{s}=1015^{\circ} \mathrm{C}$ and at $T_{\mathrm{c}}=1000^{\circ} \mathrm{C}$. In Fig. 6 abscissa (I) represents $\mathrm{Zn}$ partial pressure $P_{\mathrm{zn}}$, abscissa (2) $P_{\mathrm{Se}_{2}}$ in equilibrium with $P_{\mathrm{zn}}$ of abscissa (1) at $T_{s}=1015^{\circ} \mathrm{C}$, and abscissa (3) $P_{\mathrm{Se}_{2}}$ in equilibrium with $P_{\mathrm{Zn}}$ of abscissa (1) at $T_{c}=1000^{\circ} \mathrm{C}$. It is shown that the total pressure has a minimum value at a certain partial pressure at which the vapor phase composition is stoichiometric, namely $P_{\mathrm{Zn}}=2 P_{\mathrm{Se}_{2}}$, e.g., at $P_{\mathrm{Zn}}=7.07 \times 10^{-4} \mathrm{~atm}$. and $P_{\mathrm{Se}_{2}}=3.54 \times 10^{-4} \mathrm{~atm}$. for $T_{\mathrm{c}}=1000^{\circ} \mathrm{C}$.

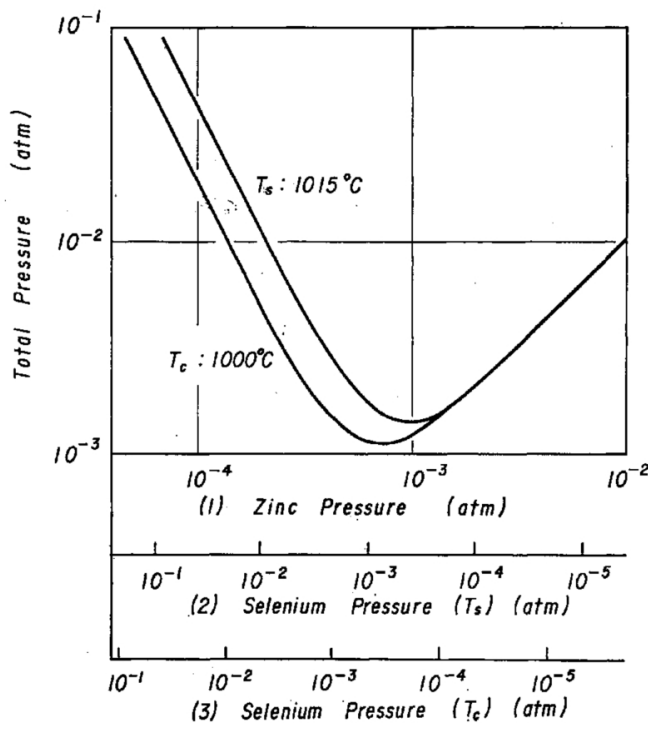

Fig. 6 Relation between total pressure and partial pressures of $\mathrm{Zn}$ and $\mathrm{Se}_{2}$ in equilibrium with solid $\mathrm{ZnSe}$ at 1015 and $1000^{\circ} \mathrm{C}$.

The partial pressure difference $\Delta P_{\mathrm{zn}}$ and $\Delta P_{\mathrm{Se}_{2}}$ between the source chamber and the growth chamber is calculated as in the following under the condition of constant total pressure over the whole region of the ampule. In the case of the $\mathrm{Zn}$ reservoir the total pressure $P$ is supposed to be determined by putting $K_{p}(T)=K_{p}\left(T_{s}\right)$ in eq. (19). On the other hand, the total pressure $P$ in the region of $T_{c}$ is expressed approximately by a lst order expansion of eq. (19) about $P_{\mathrm{Zn}}$ and equated with that at $T_{s}$;

(9) P. Goldfinger and M. Jeunehomme : Trans. Faraday Soc., $59(1963), 2851$.

$$
\begin{aligned}
P & =P_{\mathrm{zn}}+\left\{K_{p}\left(T_{s}\right) / P_{\mathrm{Zn}_{\mathrm{n}}}^{2}\right\} \\
& =\left[P_{\mathrm{Zn}_{\mathrm{n}}}+\left\{K_{p}\left(T_{c}\right) / P_{\mathrm{Zn}_{\mathrm{n}}}^{2}\right\}\right]+\left[1-\left\{2 K_{p}\left(T_{c}\right) / P_{\mathrm{Zn}_{\mathrm{n}}}^{3}\right\}\right] \Delta P_{\mathrm{zn}_{\mathrm{n}}}
\end{aligned}
$$

Then

$\Delta P_{\mathrm{Zn}}=-\Delta P_{\mathrm{Se}_{2}}=P_{\mathrm{Zn}}\left\{K_{p}\left(T_{s}\right)-K_{p}\left(T_{c}\right)\right\} /\left\{P_{\mathrm{Zn}}^{3}-2 K_{p}\left(T_{c}\right)\right\}$.

For the Se reservoir the following relation is obtained in a similar way

$$
\begin{aligned}
& \Delta P_{\mathrm{Zn}}=-\Delta P_{\mathrm{Se}_{2}=K_{p}^{1 / 2}\left(T_{s}\right)\left\{K_{p}\left(T_{s}\right)-K_{p}\left(T_{c}\right)\right\}} P_{\mathrm{Se}_{2}} / \\
&\left\{K_{p}^{3 / 2}\left(T_{s}\right)-2 K_{p}\left(T_{c}\right) P_{\mathrm{Se}_{2}}^{3 / 2}\right\}
\end{aligned}
$$

Assuming that the partial pressure gradient is uniform between the source chamber and the growth chamber and the distance between them is $l, \partial P_{1} / \partial x=-\partial P_{2} / \partial x$ leads to $\Delta P_{\mathrm{Zn}} / l=-\Delta P_{\mathrm{Se}_{2}} / l$.

The final expressions for the transport rate is written as in the following eqs. (24) and (25) for the $\mathrm{Zn}$ reservoir and the Se reservoir, respectively.

$$
\begin{aligned}
\Psi_{\mathrm{ZnSe}}(\mathrm{g} / \text { day })= & A^{\prime \prime} P_{\mathrm{Zn}}\left\{K_{p}\left(T_{s}\right)-K_{p}\left(T_{c}\right)\right\} /\left\{\left(P_{\mathrm{zn}} / 2\right)-P_{\mathrm{Se}_{2}}\right\} \\
& \left\{P_{\mathrm{Zn}}^{3}-2 K_{p}\left(T_{c}\right)\right\}
\end{aligned}
$$

and

$$
\begin{gathered}
\Psi_{\mathrm{ZnSe}}(\mathrm{g} / \mathrm{day})=A^{\prime \prime} K_{p}^{1 / 2}\left(T_{s}\right)\left\{K_{p}\left(T_{s}\right)-K_{p}\left(T_{c}\right)\right\} P_{\mathrm{Se}_{2}} / \\
\left\{\left(P_{\mathrm{Zn}} / 2\right)-P_{\mathrm{Se}_{2}}\right\}\left\{K_{p}^{3 / 2}\left(T_{s}\right)-2 K_{p}\left(T_{c}\right) P_{\mathrm{Se}_{2}}^{3 / 2}\right\}
\end{gathered}
$$

where $A^{\prime \prime}=227.1 \times S W\left\{T^{\prime 3}\left(M_{1}+M_{2}\right) / 2 M_{1} M_{2}\right\}^{1 / 2} /$ $l N_{0} k T^{\prime}\left\{\left(\sigma_{1}+\sigma_{2}\right) / 2\right\}^{2}$,

$l$ is a distance between the source chamber and the growth chamber and $S$ a cross sectional area of the ampule.

From these equations, some characteristic partial pressure dependence of the transport rate is foreseen.

(1) In case of the $\mathrm{Zn}$ reservoir $\left\{\right.$ eq. (24)\}, if $P_{\mathrm{Zn}_{\mathrm{n}}}$ is high and predominant in total pressure $P, \Psi_{\mathrm{znSe}}$ is proportional to $P_{\mathrm{Zn}}^{-3}$, and if $P_{\mathrm{Zn}}$ is low and $P_{\mathrm{Se} 2}=K_{p}\left(T_{s}\right) /$ $P_{\mathrm{Zn}}^{2}$ is predominant in total pressure $P, \Psi_{\mathrm{znSe}}$ is proportional to $P_{\mathrm{Zn}}^{3}$.

(2) In case of Se reservoir $\left\{\right.$ eq. (25)\}, if $P_{\mathrm{Se}_{2}}$ is high and predominant in total pressure $P, \Psi_{\mathrm{ZnS} \theta}$ is proportional to $P_{\mathrm{Se}_{2}}^{-3 / 2}$, and if $P_{\mathrm{Se}_{2}}$ is low and $P_{\mathrm{Zn}}=\left\{K_{p}\left(T_{s}\right) \mid\right.$ $\left.P_{\mathrm{Se}_{2}}\right\}^{1 / 2}$ is predominant in total pressure $P, \Psi_{\mathrm{ZnSe}}$ is proportional to $P_{\mathrm{Se}_{2}}^{3 / 2}$.

It should be noticed that eqs. (24) and (25) become rough approximations near the minimum total pressure and cannot be applied for this pressure.

The results calculated from eqs. (24) and (25) are shown in Figs. 2 and 3, respectively. Values of the $\mathrm{Zn}$ gas molecular diameter are derived from the viscosity coefficient data and estimated as $3.01 \mathrm{~A}$ and $2.97 \mathrm{~A}$ for $T^{\prime}=1008^{\circ} \mathrm{C}$ and $1108^{\circ} \mathrm{C}$, respectively. Concerning the molecular diameter of $\mathrm{Se}_{2}$, those of $\mathrm{Br}_{2}$ gas molecule estimated from viscosity data as $4.52 \mathrm{~A}$ and $4.48 \mathrm{~A}$ for the respective temperatures are used since the gas molecular diameter of $\mathrm{O}_{2}$ is similar to that of $\mathrm{F}_{2}{ }^{(10)}$.

From the comparison between experimental and calculated results, the following points become clear :

(1) In either case of the $\mathrm{Zn}$ of Se reservoir, when

(10) Landolt-Boernstein : Zahlenwerte und Funktionen I, 1 (1950), 325, 369. 
$P_{\mathrm{Zn}}$ or $P_{\mathrm{Se}_{2}}$ determined by the $\mathrm{Zn}$ or Se reservoir temperature is much higher than $P_{\mathrm{Zn}}$ or $P_{\mathrm{Se}_{2}}$ at the minimum total pressure, the agreement between the experimental and calculated results is good.

(2) On the contrary, when $P_{\mathrm{Zn}}$ or $P_{\mathrm{Se}_{2}}$ determined by the respective reservoir temperatures is lower than $P_{\mathrm{zn}}$ or $P_{\mathrm{Se}_{2}}$ at the minimum total pressure, the experimental results do not show such partial pressure dependence as calculated and is nearly constant over a wide range of partial pressure.

The correspondence between the experimental and theoretical results shows a quite different feature in the two partial pressure regions divided by the boundary corresponding to the minimum total pressure. This fact makes it possible to elucidate the situation realized in the ampule and to discuss the possibility of partial pressure control as used in this study. That is,

(1) In the high partial pressure region in which good agreement between the experimental and calculated results is obtained, the assumptions (1) and (2) about the situation in the ampule and consequently the partial pressure control by the $\mathrm{Zn}$ or Se reservoir are realized.

(2) In the low partial pressure region with a discrepancy between experimental and calculated results, the assumptions (1) and (2) and consequently the partial pressure control by the Zn or Se reservoir, are no longer realized, and another mechanism different from these assumptions becomes rate-determining. As an example, let us consider the case of $\mathrm{Zn}$ reservoir. When $P_{\mathrm{Zn}}$ determined by $T_{\mathrm{Zn}}$ is lower than that in congruent vaporization of solid $\mathrm{ZnSe}$, the $P_{\mathrm{Se}_{2}}$ which should be in equilibrium with the $P_{\mathrm{Zn}}$ will become very high. Such a preferential evaporation of $\mathrm{Se}$ atoms from solid $\mathrm{ZnSe}$ seems difficult, and even if possible, the dew point for the $P_{\mathrm{Se}_{2}}$ lies between $T_{s}$ and $T_{\mathrm{zn}}$ and the condensation of $\mathrm{Se}_{2}$ vapor occurs somewhere in the ampule. This makes the $\mathrm{Zn}$ reservoir ineffective for controlling $P_{\mathrm{zn}}$.

The experimental result shows that the transport rate become constant in the partial pressure region lower than that corresponding to the minimum total pressure. Therefore, it can be predicted that some situation similar to that of the minimum total pressure is realized in this region. Similar consideration may be applicable to the case of the Se reservoir.

Therefore, the following conditions are proposed for the $\mathrm{ZnSe}$ single crystal growth by the sublimation method with the $\mathrm{Zn}$ or Se reservoir.

(1) Rapid growth of large single crystals requires lower $T_{\mathrm{zn}}$ or $T_{\mathrm{Se}}$ which gives lower $P_{\mathrm{zn}}$ or $P_{\mathrm{Se}_{2}}$ than that corresponding to the minimum total pressure (stoichiometric vapor phase composition).

(2) In order to realize partial pressure control by using the $\mathrm{Zn}$ or Se reservoir, higher $T_{\mathrm{zn}}$ or $T_{\mathrm{Se}}$ is required which gives higher $P_{\mathrm{zn}}$ or $P_{\mathrm{Se}_{2}}$ than that corresponding to the minimum total pressure.

At the same time this condition seems to be required for controlling of the deviation from stoichiometry in the grown crystals.

\section{Summary}

ZnSe single crystals were grown by the sublimation method under controlled partial vapor pressure of the constituent elements in a closed ampule with the $\mathrm{Zn}$ or Se reservoir. The following results were obtained :

(1) The vapor phase in equilibrium with solid $\mathrm{ZnSe}$ has the minimum total pressure at a certain value of each partial pressure at which the vapor phase has stoichiometric composition. When $\mathrm{Zn}$ or $\mathrm{Se}_{2}$ partial pressure calculated from the $\mathrm{Zn}$ or Se reservoir temperature is lower than that corresponding to the minimum total pressure, the transport rate of $\mathrm{ZnSe}$ crystals from the source chamber to the crystal growth chamber via the vapor phase is independent of $\mathrm{Zn}$ or $\mathrm{Se}_{2}$ partial pressure, and is nearly constant over a wide partial vapor pressure region. On the other hand, when $\mathrm{Zn}$ or $\mathrm{Se}_{2}$ partial pressure is higher than that corresponding to the minimum total pressure, the transport rate is proportional to $-3 \mathrm{rd}$ power of $\mathrm{Zn}$ partial pressure and to the $-3 / 2$ th power of $\mathrm{Se}_{2}$ partial pressure.

(2) The experimental results of the transport rate were compared with those calculated based on the assumption that the rate determining process for the mass transport phenomenon is the diffusion in the vapor phase. In the region of higher $\mathrm{Zn}$ or $\mathrm{Se}_{2}$ partial pressure than that corresponding to the minimum total pressure, good agreement is obtained between observation and calculation, but in the lower $\mathrm{Zn}$ or $\mathrm{Se}_{2}$ partial pressure region, a large discrepancy is observed.

(3) Koňák et al. (4) and Toyama (11) have reported and discussed about the single crystal growth of some kinds of $\mathrm{A}^{\mathrm{II}} \mathrm{B}^{\mathrm{VI}}$ compounds under controlled excess partial pressure of one of the constituent elements, but in a manner different from that of the present study. According to them, such a situation is considered inside the crystal growth ampule that the congruent evaporation occurs under excess partial vapor pressure and the rate determining process for the mass transport is also the diffusion in the vapor phase. They also reported the partial pressure dependence of the transport rate on the $-3 \mathrm{rd}$ power of excess partial pressure of the II group element and the $-3 / 2$ th power of excess partial pressure of the VI group element, in agreement with our calculation. They did not carry out any experiments for the low partial pressure, and their comparison between the experimental and calculated results is unsatisfactory. Also, the results of numerical calculations by Toyama ${ }^{(11)}$ do not agree with our experimental results.

In the present study the experimental and calculated results in a wide partial pressure range were compared. In the higher partial pressure region a fairly good agreement was obtained and at the same time the partial pressure region where the assumptions about the situation inside a growth ampule are valid was made clear.

(4) The rapid growth of large single crystals requires lower $T_{\mathrm{zn}}$ or $T_{\mathrm{Se}}$ which gives lower $P_{\mathrm{zn}}$ or $P_{\mathrm{Se}_{2}}$ than that corresponding to the minimum total pressure. On 
the other hand, higher $T_{\mathrm{zn}}$ or $T_{\mathrm{Se}}$ is necessary in order to realize the control of partial pressure and, therefore, of the deviation from stoichiometry in the grown crystals.

(5) The grown crystals have always a crystal structure of the zinc blende type and their typical shapes are columnar with a nearly equilateral hexagonal cross section surrounded by six $\{110\}$ planes grown in one of the $\langle 111\rangle$ directions and plate-like grown in two of the $\langle 111\rangle$ directions.

Two types of twin structure were found out; one due to growth faults in only one of the $<111>$ directions which corresponds to the growth direction of the columnar crystals and the other due to the growth faults in at least two of the $\langle 111\rangle$ directions which correspond to growth directions of the plate-like crystals.

\section{Acknowledgment}

The authors are indebted to Dr. H. Kojima of Research Institute for Scientific Measurements for his kindness on performing the work of oscillating crystals and Weissenberg X-ray diffractometry. 